\title{
A Study of Cognitive Dysfunctions in Type 2 Diabetes Mellitus
}

\author{
Sandeep Alex ${ }^{1}$ \\ ${ }^{1}$ Department of Psychiatry, Government TD Medical College, Alappuzha, Kerala, India.
}

\section{ABSTRACT}

\section{BACKGROUND}

Diabetes mellitus (DM) refers to a group of common metabolic disorders that share the phenotype of hyperglycaemia. The worldwide prevalence of DM has dramatically increased over the past 2 decades. The prevalence of DM increases with age from $0.19 \%$ in persons below 20 years to $20.1 \%$ in those above 65 years. Type 2 diabetes mellitus is known to produce macro vascular complications. Now compelling evidence is emerging about the effect of diabetes on the brain and resultant cognitive dysfunctions. Against this backdrop, we set out to study the cognitive dysfunctions in type 2 diabetes mellitus, since the presence of cognitive dysfunctions may adversely affect management of diabetes, which would have important clinical and public health implications.

\section{METHODS}

This is a cross sectional study done in a tertiary care teaching hospital. Institutional ethical committee approved the study. Informed consent was taken from the study participants. 30 in-patients with type 2 diabetes mellitus were matched against 30 non-diabetic controls. The sample was matched for age, gender, education and socio economic status. Demographic details of the subjects were collected using the sociodemographic and clinical data proforma designed especially for the study. The tools used were Standardized Mini Mental State Examination (SMMSE) and Brief Cognitive Rating Scale (BCRS). Data was analysed using Student's t-test, Chi-square test, ANOVA and Mann Whitney U test.

\section{RESULTS}

The mean score on SMMSE obtained by patients with type 2 diabetes was 24.26 while that obtained by comparator normoglycemic group was 28.06 and this difference was very highly significant ( $p$ value 0.001 ). However, the lower mean total score obtained by the diabetics on SMMSE (24.26), though statistically very significant with respect to the non-diabetic comparator group, was still within the normative data, which needs further analysis. In BCRS, very highly significant difference ( $p$ value 0.001) between the two groups was found with diabetics performing poorly in concentration, recent memory, past memory, and orientation domains while no significant difference was found in the functioning and self-care domain. Longer duration of type 2 diabetes led to poorer performance on SMMSE. This is reflected in the mean scores obtained by patients, with those with duration of illness greater than 10 years having a mean score of 23.77 while those with less than 5 years of type $2 \mathrm{DM}$ achieving mean score of 27.36. Poorly controlled type 2 DM as indicated by higher HbA1c values affected SMMSE scores adversely with those with $\mathrm{HbA1c}$ greater than $10.3 \%$ getting a mean score of 23.63 compared to the mean score of 25.40 obtained by those with HbA1c of $6.3-8.3 \%$ and this was statistically highly significant.

\section{CONCLUSIONS}

Diabetics had significant cognitive dysfunction which accentuated further with longer duration of illness, elevated HbA1c levels, use of both Oral Hypoglycaemic Agents and Insulin and advancing age. However, the degree of cognitive dysfunctions did not endanger patient's ability to self-manage their illness.

\section{KEY WORDS}

Cognitive Dysfunctions, Type 2 Diabetes Mellitus, HbA1c, SMMSE, BCRS
Corresponding Author: Dr. Sandeep Alex, \#117-E/VII, Anaiah, Whitefield Residence, Kudamaloor, Kottayam-686017, Kerala, India.

E-mail: drsandeepalex@yahoo.co.in

DOI: $10.14260 / \mathrm{jemds} / 2019 / 835$

Financial or Other Competing Interests: None.

How to Cite This Article:

Alex S. A study of cognitive dysfunctions in type 2 diabetes mellitus. J. Evolution Med. Dent. Sci. 2019;8(51):3853-3857, DOI: 10.14260/jemds/2019/835

Submission 24-10-2019, Peer Review 05-12-2019, Acceptance 11-12-2019, Published 23-12-2019.

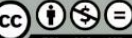




\section{BACKGROUND}

Diabetes mellitus (DM) represents a group of common metabolic disorders that share the phenotype of hyperglycaemia. Several types of DM exist and are caused by a complex interaction of genetics, environmental factors and lifestyle choices. The metabolic dysregulation associated with DM causes secondary pathophysiological changes in multiple organ systems.

The worldwide prevalence of DM has increased over the past 2 decades. The prevalence of DM is estimated to be $0.19 \%$ in persons below 20 years and $8.6 \%$ in those above 20 years. In individuals above 65 years the prevalence is $20.1 \%{ }^{1}$ Of particular concern is the realization that lifestyle changes are beginning to transform type $2 \mathrm{DM}$ from a largely adult disorder into one affecting teenagers and children.2,3 Impact of metabolic dysregulation brought by type 2 diabetes on cardiovascular health has been the major focus of researchers till now. Until recently, little consideration was given to the possibility that metabolic dysregulation might also influence brain function. But now numerous studies have shown positive association between diabetes and cognitive impairment. ${ }^{4,5}$

However, this evidence for cognitive dysfunction in type 2 DM can be accepted only with certain caveats, because it is a complex disorder. In addition to intrinsic abnormalities such as hyperglycaemia, patients with type 2 DM frequently develop multisystem complication such as retinopathy, nephropathy and neuropathy. The resulting impaired vision, renal impairment and diminished hand-eye co-ordination could all interfere with an individual's performance on psychometric testing. Patients with type 2 DM treated with insulin or sulfonylureas may also develop recurrent episodes of hypoglycaemia that are arguably associated with modest permanent cognitive dysfunctions. A range of other disorders which may be associated with cognitive dysfunctions such as hypertension, ischemic heart disease, hyperlipidaemia and depression are often co-morbid with type 2 DM. Thus, the cognitive impairment observed in type 2 DM may result from the interactions between abnormalities intrinsic to diabetes, diabetes specific complications and other diabetes related disorders. This study attempts to find out cognitive dysfunctions intrinsic to diabetes and has public health importance because impaired cognitive function is known to adversely affect drug compliance, thereby further worsening diabetes and quality of life.

We wanted to determine and compare the cognitive dysfunctions, in type 2 diabetes mellitus patients and in nondiabetics matched for age, education and socio-economic status.

\section{METHODS}

This is a cross-sectional, comparative study. Subjects for the study were selected from the in-patient admissions made under various specialities at Father Muller Medical College, a tertiary care teaching hospital in Mangalore.

\section{Sampling Technique}

After obtaining institutional ethical committee clearance, 30 patients who were diagnosed as having type 2 diabetes and 30 non-diabetic controls, matched to age, gender, education and socio-economic status were chosen by convenience sampling. The control group was selected from relatives of the patients admitted under various specialties.

\section{Sampling Procedure}

Initial contact was made in the hospital wards and suitable subjects satisfying the inclusion and exclusion criteria were identified. An informed consent was obtained from those who were willing to participate in the study. The purpose of the study was explained to the participants and they were informed that refusal to participate, would not affect the ongoing treatment or outcome adversely. Demographic details of the subjects were collected using the sociodemographic and clinical data proforma designed especially for the study. Cognitive functions of subjects chosen for the study were assessed using Standardized Mini-Mental State Examination (SMMSE) and Brief Cognitive Rating Scale (BCRS). Investigations like haemogram, random blood sugar, fasting blood sugar, post prandial blood sugar, glycosylated haemoglobin, lipid profile, liver function tests, renal function tests and ECG which were available were recorded.

\section{Inclusion Criteria for Cases}

Patients with type 2 diabetes in the age group of 30-65 years, with a minimum education level of $8^{\text {th }}$ standard, as diagnosed by the Department of Internal Medicine, based on blood sugar estimations as per World Health Organisation recommendation of Fasting Blood Sugar $\geq 126 \mathrm{mg} / \mathrm{dL}$ and 2hours post prandial blood sugar $\geq 200 \mathrm{mg} / \mathrm{dL}$.

\section{Exclusion Criteria for Cases}

Type 2 diabetics more than 65 years of age were excluded. Similarly, patients with chronic diseases which may cause cognitive impairment such as hypertension, neurodegenerative diseases like dementia and organic mental disorders, those with history of substance abuse, with past or current history of psychiatric disorders were also excluded.

\section{Inclusion Criteria for Controls}

Non-diabetic subjects in the age group of 30-65 years with a minimum education level of $8^{\text {th }}$ standard were included as controls.

\section{Exclusion Criteria for Controls}

Patients with chronic diseases which may cause cognitive impairment such as hypertension, neurodegenerative diseases like dementia and organic mental disorders, those with history of substance abuse, with past or current history of psychiatric disorders were excluded.

\section{Standardized Mini Mental State Examination (SMMSE) ${ }^{6}$}

The SMMSE is a bed-side screening test for cognitive impairment, derived from the Mini-Mental State Examination (MMSE) ${ }^{7}$, the most widely used instrument to measure cognitive impairment. SMMSE has attempted to build on the advantages of MMSE namely ease of administration and scoring while addressing its short comings viz. intra-rater 
and inter-rater variance. SMMSE is administered by a trained rater or clinician and takes about 5 - 10 minutes. It contains 12 items which are asked in sequence and generate a total score of 30 . The scores of less than 18 indicate severe cognitive impairment while $18-23$ point to mild impairment and scores above 24 is reflective of no impairment. SMMSE has clearer instructions than MMSE and has time limit for the answers, commensurate with the cognitive tasks. It has good content and concurrent validity and is organized into discrete subsections measuring orientation, registration, attention and concentration, recall, language and construction. But the subsections and individuals items cannot be viewed as measures of specific aspects of cognition, because factor analytic studies typically yield a two factor solution. Because of the non-specific nature of the individual subsection scores of SMMSE, it should be followed by a more comprehensive assessment, as attempted in the current study.

\section{Brief Cognitive Rating Scale (BCRS) ${ }^{\mathbf{8}}$}

BCRS is designed specifically to assess the syndrome of cognitive decline. As a clinical rating instrument, BCRS merges the judgment and skill of the clinician with objective rating criteria. Consequently, stable data can be obtained on subjects with cognitive impairment who may be only variably co-operative and attentive. BCRS assesses the magnitude of cognitive impairment on 5 clinical axes, each scored on a Likert scale of 1 to 7 using specified criteria. The axes represented are concentration, recent memory, past memory, orientation and functioning/ self-care. Items are scored on the basis of a structured clinical interview conducted in the presence of primary care-giver.

\section{Statistical Analysis}

The data was analyzed for statistical significance by using chisquare test for categorical variables, ANOVA, Student's t-test and Mann-Whitney $U$ test for continuous numerical variables. Carl Pearson's coefficient of correlation was used to find out the correlation between the variables.

\section{RESULTS}

A total of 60 subjects comprising of 30 patients diagnosed as having type $2 \mathrm{DM}$ and 30 non-diabetic controls were included in the study. There is no significant difference between the age of control group and cases. There was significant difference between the mean BMI value of the controls and cases $(p=0.012<0.05)$. Results indicated that diabetics were significantly overweight with respect to their non diabetic counterparts. As expected, there was very high significant difference between random blood sugar values between the two groups.

In SMMSE, the mean total score obtained by the control group was 28.06 with a standard deviation of 1.05 while that obtained by the cases group was 24.27 with a standard deviation of 1.11. The difference between the two groups was very highly significant. Results indicated circumscribed areas of cognitive dysfunction in type 2 diabetic patients with respect to controls. Table 3 depicts data on BCRS which show significant impairment in the diabetic patients with respect to controls except in the functioning and self-care domain.
The performance of patients type 2 diabetes mellitus showed that longer duration of diabetes and higher $\mathrm{HbA}_{1} \mathrm{c}$ values led to worse performance in SMMSE and BCRS as shown in table 4 and table 5 respectively.

\begin{tabular}{|c|c|c|c|c|c|}
\hline & Group & $\mathbf{N}$ & Mean & Std. Deviation & Z \\
\hline \multirow{2}{*}{ RBS } & Control & 30 & 112.2000 & 9.21543 & 5.4121 \\
\cline { 2 - 5 } & Cases & 30 & 278.1667 & 89.91724 & $\mathrm{p}=0.001$ vhs \\
\hline \multicolumn{6}{|c|}{ Table 1. Random Blood Sugar } \\
\hline Z = Mann - Whitney U test
\end{tabular}

\begin{tabular}{|c|c|c|c|c|}
\hline & Group & Mean & Std. Deviation & $\mathbf{t}$ \\
\hline \multirow{2}{*}{ Orientation } & Control & 9.3000 & 0.59596 & \multirow{2}{*}{$\begin{array}{c}6.62500 \\
p=0.001 \mathrm{vhs}\end{array}$} \\
\hline & Case & 8.3667 & 0.49013 & \\
\hline \multirow{2}{*}{ Registration } & Control & 3.0000 & 0.00000 & \multirow{2}{*}{$\begin{array}{c}6.59500 \\
p=0.001 \mathrm{vhs}\end{array}$} \\
\hline & Case & 2.4000 & 0.49827 & \\
\hline \multirow{2}{*}{ Attention } & Control & 4.2000 & 0.61026 & \multirow{2}{*}{$\begin{array}{c}9.41400 \\
p=0.001 \mathrm{vhs}\end{array}$} \\
\hline & Case & 2.7667 & 0.56832 & \\
\hline \multirow{2}{*}{ Recall } & Control & 2.6667 & 0.47946 & \multirow{2}{*}{$\begin{array}{c}5.45300 \\
\mathrm{p}=0.001 \mathrm{vhs}\end{array}$} \\
\hline & Case & 2.0667 & 0.36515 & \\
\hline \multirow{2}{*}{ Language } & Control & 8.0000 & 0.00000 & \multirow{2}{*}{0.00000} \\
\hline & Case & 8.0000 & 0.00000 & \\
\hline \multirow{2}{*}{ Construction } & Control & 0.8333 & 0.37905 & \multirow{2}{*}{$\begin{array}{c}1.76800 \\
p=0.082 \mathrm{~ns}\end{array}$} \\
\hline & Case & 0.6333 & 0.49013 & \\
\hline \multirow{2}{*}{ Total Score } & Control & 28.0667 & 1.04826 & \multirow{2}{*}{$\begin{array}{c}13.61900 \\
p=0.001 \text { vhs }\end{array}$} \\
\hline & Case & 24.2667 & 1.11211 & \\
\hline
\end{tabular}

Table 2. Standardized Mini - Mental State Examination t-Student's t-test

\begin{tabular}{|c|c|c|c|c|}
\hline & Group & Mean & Std. Deviation & $\mathbf{t}$ \\
\hline \multirow{2}{*}{ Concentration } & Control & 1.4333 & 0.50401 & \multirow{2}{*}{$\begin{array}{c}8.70900 \\
p=0.001 \mathrm{vhs}\end{array}$} \\
\hline & Case & 2.5667 & 0.50401 & \\
\hline \multirow{2}{*}{ Recent memory } & Control & 1.2333 & 0.43018 & \multirow{2}{*}{$\begin{array}{c}5.24200 \\
\mathrm{p}=0.001 \mathrm{vhs}\end{array}$} \\
\hline & Case & 1.8000 & 0.40684 & \\
\hline \multirow{2}{*}{ Past memory } & Control & 1.0000 & 0.00000 & \multirow{2}{*}{$\begin{array}{c}5.75700 \\
p=0.001 \mathrm{vhs}\end{array}$} \\
\hline & Case & 1.5333 & 0.50742 & \\
\hline \multirow{2}{*}{ Orientation } & Control & 1.0000 & 0.00000 & \multirow{2}{*}{$\begin{array}{c}2.11200 \\
p=0.039 \mathrm{sig}\end{array}$} \\
\hline & Case & 1.2667 & 0.69149 & \\
\hline \multirow{2}{*}{$\begin{array}{l}\text { Functioning and } \\
\text { self-care }\end{array}$} & Control & 1.0000 & 0.00000 & \multirow{2}{*}{$\begin{array}{c}1.79500 \\
\mathrm{p}=0.078 \mathrm{~ns}\end{array}$} \\
\hline & Case & 1.1000 & 0.30513 & \\
\hline \multirow{2}{*}{ Total score } & Control & 1.1333 & 0.13218 & \multirow{2}{*}{$\begin{array}{c}7.49500 \\
p=0.001 \mathrm{vhs}\end{array}$} \\
\hline & Case & 1.6467 & 0.35109 & \\
\hline \multicolumn{5}{|c|}{ Table 3. Brief Cognitive Rating Scale } \\
\hline t-Stuo & & & & \\
\hline
\end{tabular}

\begin{tabular}{|c|c|c|c|c|c|}
\hline & Duration in Years & Mean Score & S.D. & $\mathbf{F}$ & $\mathbf{p}$ \\
\hline \multirow{3}{*}{ SMMSE } & $<5$ & 27.3684 & 1.74636 & \multirow{3}{*}{32.352} & \multirow{3}{*}{$0.001 \mathrm{vhs}$} \\
\hline & 5-10 & 24.3077 & 1.03155 & & \\
\hline & $>10$ & 23.7778 & 0.97193 & & \\
\hline \multirow{3}{*}{ BCRS } & $<5$ & 1.2000 & 0.20795 & \multirow{3}{*}{26.969} & \multirow{3}{*}{$0.001 \mathrm{vhs}$} \\
\hline & $5-10$ & 1.6462 & 0.34789 & & \\
\hline & $>10$ & 1.8222 & 0.36667 & & \\
\hline
\end{tabular}

Table 4. Duration of diabetes mellitus vs Cognitive Tests (Cases)

\begin{tabular}{|c|c|c|c|c|c|}
\hline & HbA1c\% & Mean Score & Std. Deviation & $\mathbf{F}$ & $\mathbf{p}$ \\
\hline \multirow{3}{*}{ SMMSE } & $6.3-8.3$ & 25.4000 & 0.54772 & \multirow{3}{*}{5.899} & \multirow{3}{*}{$0.007 \mathrm{hs}$} \\
\hline & $8.3-10.3$ & 24.3571 & 1.08182 & & \\
\hline & $>10.3$ & 23.6364 & 0.92442 & & \\
\hline \multirow{3}{*}{ BCRS } & $6.3-8.3$ & 1.3200 & 0.10954 & \multirow{3}{*}{5.535} & \multirow{3}{*}{$0.01 \mathrm{hs}$} \\
\hline & $8.3-10.3$ & 1.6000 & 0.27175 & & \\
\hline & $>10.3$ & 1.8545 & 0.39080 & & \\
\hline \multicolumn{6}{|c|}{ Table 5: HbA1c \% vs Cognitive Tests (Cases) } \\
\hline
\end{tabular}

\section{DISCUSSION}

Changes in cognitive ability in old age are brought about both by processes of 'normal' ageing and by a steadily accumulating burden of pathologies. The main task of cognitive gerontology is to compare the relative contributions of these two factors and their interactions. 
This study has tried to address these issues by investigating cognitive functions in type $2 \mathrm{DM}$ patients matched against non-diabetic controls with respect to age, gender, education and socio-economic status. The study revealed that there is statistically significant cognitive impairment in type 2 diabetes patients compared with controls which persisted even after correction for confounding factors. Cognitive tests were administered to the subjects within 2 hours after the last meal ingestion. This precaution was taken because type 2 DM patients are prone to develop discrete episodes of hypoglycaemia, as an adverse effect of medications prescribed to achieve normoglycemia. Since hypoglycaemia is known to affect cognitive functions adversely, cognitive testing during such hypoglycaemic episodes might lead to spurious results.

There was statistically significant difference between the body mass index of diabetics and non-diabetics in the current study. This was in agreement with results from a previous study. ${ }^{9}$ However, there was an essential difference. The mean BMI (23.41) of diabetics in the current study was still within normal limits (BMI: 18-24) unlike in many studies in which BMI of diabetic patients was in the range of obesity. This assumes importance because of the findings by Elias et al 10 from the Framingham investigation of obesity and cognitive function, identified obesity as a risk factor for cognitive dysfunction independently of diabetes, total cholesterol, alcohol consumption, cigarette smoking, hypertension and stroke. The fact that, in the current study, diabetic subjects were not obese eliminated a potential confounding factor and gave further credence to the hypothesis at the beginning of the study, i.e., cognitive deficits observed are due to the intrinsic effect of diabetes.

A host of studies have found impairments in MMSE in diabetic patients. ${ }^{9,11,12}$ In contrast, few studies did not find significant difference between diabetics and non-diabetics in their performance on MMSE 13,14In this context, what can we infer from the SMMSE scores from this study? In the past, a cut off score of less than 24 on MMSE was considered to indicate cognitive impairment, while that above 24 indicated no cognitive impairment. Interestingly, Kalijnet al ${ }^{12}$ have added one more category, that of borderline cognitive impairment, indicated by MMSE total scores of 24 and 25. In the current study diabetics obtained the mean score of 24.26, which can be interpreted in two ways depending on the cutoff score used, i.e., low normal on using the cut-off score of 24 or borderline cognitive impairment on using the cut-off score of 26.

Both of these methods are not above reproach, because SMMSE is affected by age and education. SMMSE is less than ideal when those with mild cognitive impairment are evaluated, as it might miss these subjects, which very well could have been the case with the current study. Another possibility is that, since SMMSE is biased toward verbal items and does not adequately measure other cognitive functions such as ability to attend to relevant input, ability to solve abstract problems, psychomotor speed and visuospatial ability, cognitive functions that would have been the first to be affected by diabetes. 15,16,17

This means that SMMSE should not be used in lieu of more comprehensive neuropsychological assessment of cognitive function, a major strength of the current study which assessed the subjects in further detail with BCRS.
The use of BCRS in measuring cognitive impairment in type $2 \mathrm{DM}$ is a novel strategy as hitherto no studies have employed it in their battery of neuropsychological tests. Statistical analysis revealed very highly significant impairment in the concentration, recent memory and past memory domains of BCRS which correlated with domain trends noted in SMMSE.

A major plus point of BCRS is the provision of a likert scale for assessing functioning and self-care, making it possible to assess the impact of diabetes on patient's activities of daily living (ADL) and functioning, the greatest area of concern. Current study revealed that the impact of diabetes on functioning and self-care was not significant. This is a welcome finding, since the cornerstone of successful diabetes treatment is self-management i.e., patients with diabetes are expected to observe strict drug compliance, periodically test their blood glucose, exercise, consume a healthy diet, initiate and maintain, major, often unpleasant life style changes. With due caution, diabetes patients can be reassured that, even though diabetes is associated with cognitive impairment, this decline in itself is unlikely to endanger their ability to self-manage their illness.

Glycosylated $\mathrm{Hb}$ level is a long-term index of glycaemic control. In the current study there was a highly significant inverse correlation between cognitive impairment and $\mathrm{HbA} 1 \mathrm{c} \%$ in SMMSE and BCRS. These observations are consistent with an earlier study from Japan. ${ }^{18}$ Earlier studies, ${ }^{19,20,21,22}$ dwelt on the effect of medication used to achieve normoglycemia and cognitive function. These studies evaluated the impact of oral hypoglycaemic agents (OHA) or insulin on cognitive function. Current study added one more variable of combined use of OHA as well as insulin in the statistical analysis. Results indicated that greatest reduction in cognitive function was seen in those taking both insulin and OHA followed by insulin alone and OHA alone group. Possible explanations for this include the hyper insulinemic state induced by the chronic use of insulin and the hypoglycaemic episodes which are more likely to occur with insulin use than OHA use. Other reasons for this association might be the fact that, patients taking insulin would have had longer duration of diabetes, more severe illness and are more aged. As expected, longer the duration of diabetes, greater was the impairments noted in SMMSE and BCRS which is a replication of finding from an earlier study by Croxson et al. ${ }^{11}$

However, there are certain limitations to this study. Current study was a cross-sectional one. Performance at a single point may bear little relation to subsequent changes in performance overtime. Therefore, the ability to infer change from a single observation is limited. A causal relationship cannot be established from a cross-sectional study because across-sectional relationship may be a result of associations with a third variable in common with both the risk factor and cognitive function rather than a true cause and effect.

Since cognition is a measure of change in an individual over time, longitudinal studies are more reliable. The use of more than one data point can significantly reduce random variability and increase power for detecting associations. Thus, longitudinal studies that link risk factors with direct measures of change in cognitive function over time greatly reduce bias, allow more definitive conclusions to be drawn regarding cause and effect, and markedly increase study power. But, unfortunately, they are much more costly and are 
logistically more demanding. Longitudinal studies are subject to their own sources of bias, especially the survivor bias due to the high attrition rate. In several studies, subjects with diabetes who had poor cognitive function at base line were more likely not to attend for follow-up leading to a potential underestimation of the effect of diabetes on cognitive function. The relationship between diabetes and cognitive impairment may also be blurred by a learning effect, obscuring any true underlying decline in cognitive ability. Depression, a common co morbidity of type 2 DM can confound the results. In this study depression was ruled out basing on careful clinical examination but no objective or subjective rating scales were used.

\section{CONCLUSIONS}

The genesis and pattern of cognitive deficits in the diabetic population is complex. However, it appears from this study that such deficits do exist and are associated with advancing age, longer duration of poorly controlled diabetes, higher $\mathrm{HbA}_{1} \mathrm{C}$ values and the combined use of Insulin and Oral Hypoglycaemic Agents rather than OHA alone. Further studies which evaluate other aspects of cognitive functions such as visuomotor coordination, psychomotor speed, motor persistence and mental flexibility which are likely to be affected earlier than the cognitive impairments captured in this study are required. Even modest reductions in cognitive function result in substantially increased risks of dementia over several years. Hence, prevention and control of type 2 diabetes have critical public health consequences.

\section{ACKNOWLEDGEMENT}

The author thanks Dr. K. Krishnamurthy, Former Professor and Head of the Department of Psychiatry of Fr. Muller Medical College for his guidance and encouragement.

\section{REFERENCES}

[1] Powers AC. diabetes mellitus. In: Kasper DL, Braunwald E, Fauci AS, et al. eds. Harrison's Principles of Internal Medicine. $16^{\text {th }}$ edn. New Delhi: McGraw-Hill Publications 2005: p. 2152-80.

[2] Kitagawa $\mathrm{T}$, Owaki $\mathrm{M}$, Urakami $\mathrm{T}$, et al. Increased incidence of non-insulin dependent diabetes mellitus among Japanese school children correlates with an increased intake of animal protein and fat. Clin Pract 1998;37(2):111-5.

[3] Bloomgarden ZT. type 2 diabetes in the young: the evolving epidemic. diabetes Care 2004;27(4):998-1010.

[4] Strachan MW, Deary IJ, Ewing FM, et al. Is type II diabetes associated with an increased risk of cognitive dysfunction? A critical review of published studies. diabetes Care 1997;20(3):438-45.
[5] Gregg EW, Brown A. Cognitive and physical disabilities and aging-related complications of diabetes. Clinical diabetes 2003;21(3):113-8.

[6] Molloy DW, Alemayehu E, Roberts R. Reliability of a standardized mini-mental state examination compared with the traditional mini-mental state examination. Am J Psychiatry 1991;148(1):102-5.

[7] Spreen 0, Strauss E. General ability and premorbid intelligence. In: A compendium of neuropsychological tests: administration, norms and commentary. $2^{\text {nd }}$ edn. New York: Oxford University Press 1998: p. 65-74.

[8] Reisberg B, Ferris SH. Brief Cognitive Rating Scale. Psychopharmacol Bull 1988;24(4):629-36.

[9] Vanhanen M, Koivisto K, Kuuisto J, et al. Cognitive function in an elderly population with persistent impaired glucose tolerance. diabetes Care 1998;21(3):398-402.

[10] Elias MF, Elias PK, Sullivan LM, et al. Obesity, diabetes and cognitive deficit: The Framingham Heart Study. Neurobiol Aging 2005;26(Suppl 1):S11-S16.

[11] Croxson SC, Jagger C. diabetes and cognitive impairment: a community-based study of elderly subjects. Age Ageing 1995;24(5):421-4.

[12] Kalmijn S, Feskens EJM, Launer LJ, et al. Glucose intolerance, hyperinsulinaemia and cognitive function in a general population of elderly men. Diabetologia 1995;38(9):1096-102.

[13] Muquit MM, Ferdous HS. Cognitive impairment in elderly, non-insulin dependent diabetic men in Bangladesh. Bangladesh Med Res Counc Bull 1998;24(2):23-6.

[14] Lindeman RD, Romero LJ, LaRue A, et al. A Biethnic community survey of cognition in participants with type 2 diabetes, impaired glucose tolerance and normal glucose tolerance. diabetes Care 2001;24(9):1567-72.

[15] Ryan CM, Geckle MO. Circumscribed cognitive dysfunction in middle-aged adults with type 2 diabetes. diabetes Care 2000;23(10):1486-93.

[16] Ryan CM, Williams TM, Orchard TJ, et al. Psychomotor slowing is associated with distal symmetrical polyneuropathy in adults with diabetes mellitus. diabetes 1992;41(1):107-13.

[17] Ryan CM. diabetes, aging and cognitive decline. Neurobiol Aging 2005;26(Suppl 1):S21-5.

[18] Mogi N, Umegaki H, Hattori A, et al. Cognitive function in Japanese elderly with type 2 diabetes mellitus. J diabetes Complications 2004;18(1):42-6.

[19] Ott A, Stolk RP, Hofman A, et al. Association of diabetes mellitus and dementia: the Rotterdam Study. Diabetologia 1996;39(11):1392-7.

[20] Gregg EW, Yaffe K, Cauley JA, et al. Is diabetes associated with cognitive impairment and cognitive decline among older women? Study of Osteoporotic Fractures Research Group. Arch Intern Med 2000;160(2):174-80.

[21] Logroscino G, Kang JH, Grodstein F. Prospective study of type2 diabetes and cognitive decline in women aged 7081 years. BMJ 2004;328(7439):548-51.

[22] Arvanitakis Z, Wilson RS, Biennias JL, et al. diabetes mellitus and risk of Alzheimer disease and decline in cognitive function. Arch Neurol 2004;61(5):661-6. 